Case

Report

\title{
Multiple Perivascular Epithelioid Cell Tumors: Clear Cell Tumor of the Lung Accompanied by Angiomyolipoma of the Liver
}

\author{
Shinya Neri, MD,${ }^{1}$ Genichiro Ishii, MD, ${ }^{2}$ Keiju Aokage, MD,${ }^{1}$ Tomoyuki Hishida, MD, ${ }^{1}$ \\ Junji Yoshida, MD, ${ }^{1}$ Mitsuyo Nishimura, MD, ${ }^{1}$ and Kanji Nagai, $\mathrm{MD}^{1}$
}

\begin{abstract}
Clear cell tumor (CCT) of the lung is very rare, and angiomyolipoma (AML) of the liver is also very rare. Both CCT and AML have been identified as a group of neoplasms with perivascular epithelioid cell differentiation (PEComa). We report a case with multiple PEComas of a combination of CCT of the lung and AML of the liver. The patient underwent surgical resection of an abnormal nodule of the lung 5 years after treatment of AML of the liver. The histological diagnosis of the pulmonary nodule was CCT. Neither lesion demonstrated malignant phenotypes, such as high mitotic activity, necrosis, or lymphovascular invasion. Each tumor of the lung and liver was solitary and differed from each other histologically. Therefore, these tumors were considered to be multifocal, not metastatic PEComas. This case is, to our knowledge, the first report of multiple PEComas of pulmonary CCT and hepatic AML. These findings suggest that patients with PEComas may require whole-body follow-up examinations because different subtypes of PEComas may occur multifocally.
\end{abstract}

Keywords: PEComa, perivascular epithelioid cell tumor, clear cell tumor, angiomyolipoma

\section{Introduction}

The World Health Organization has formally recognized a group of neoplasms with perivascular epithelioid cell (PEC) differentiation, named PEComa. ${ }^{1)}$ PEComas include clear cell tumor (CCT), lymphangioleiomyomatosis (LAM) and angiomyolipoma (AML). ${ }^{1-3)}$ We report a case of CCT of the lung with hepatic AML, which was

${ }^{1}$ Division of Thoracic Oncology, National Cancer Center Hospital East, Kashiwa, Chiba, Japan

${ }^{2}$ Pathology Division, Research Center for Innovative Oncology, National Cancer Center Hospital East, Kashiwa, Chiba, Japan

Received: October 14, 2012; Accepted: March 7, 2013

Corresponding author: Shinya Neri, MD. Division of Thoracic Oncology, National Cancer Center Hospital East, 6-5-1 Kashiwanoha, Kashiwa, Chiba 277-8577, Japan

Email: nerithoracsurg@yahoo.co.jp

(C)2014 The Editorial Committee of Annals of Thoracic and Cardiovascular Surgery. All rights reserved. considered to be multiple PEComas originating from the lung and liver.

\section{Case Report}

The patient was a 38-year-old man who underwent lung surgery in 2010. He had received a diagnosis of tuberculosis in 2000 and had regular follow-ups thereafter at a general hospital. He subsequently underwent surgical resection of an angiomyolipoma of the liver in 2005. An abnormal shadow was pointed out on a chest X-ray film in 2010, although it had not been detected in 2009. A chest computed tomography (CT) scan revealed a well-defined $1.7 \times 1.3-\mathrm{cm}$ solitary nodule in segment 4 of the right lung. (Fig. 1A). An 18F-fluoro-2 deoxy-Dglucose positron emission tomography (FDG-PET) scan showed moderate uptake in the tumor with a maximum standardized uptake value (SUVmax) of 1.9. A definitive diagnosis could not be obtained, but a benign or low-grade 

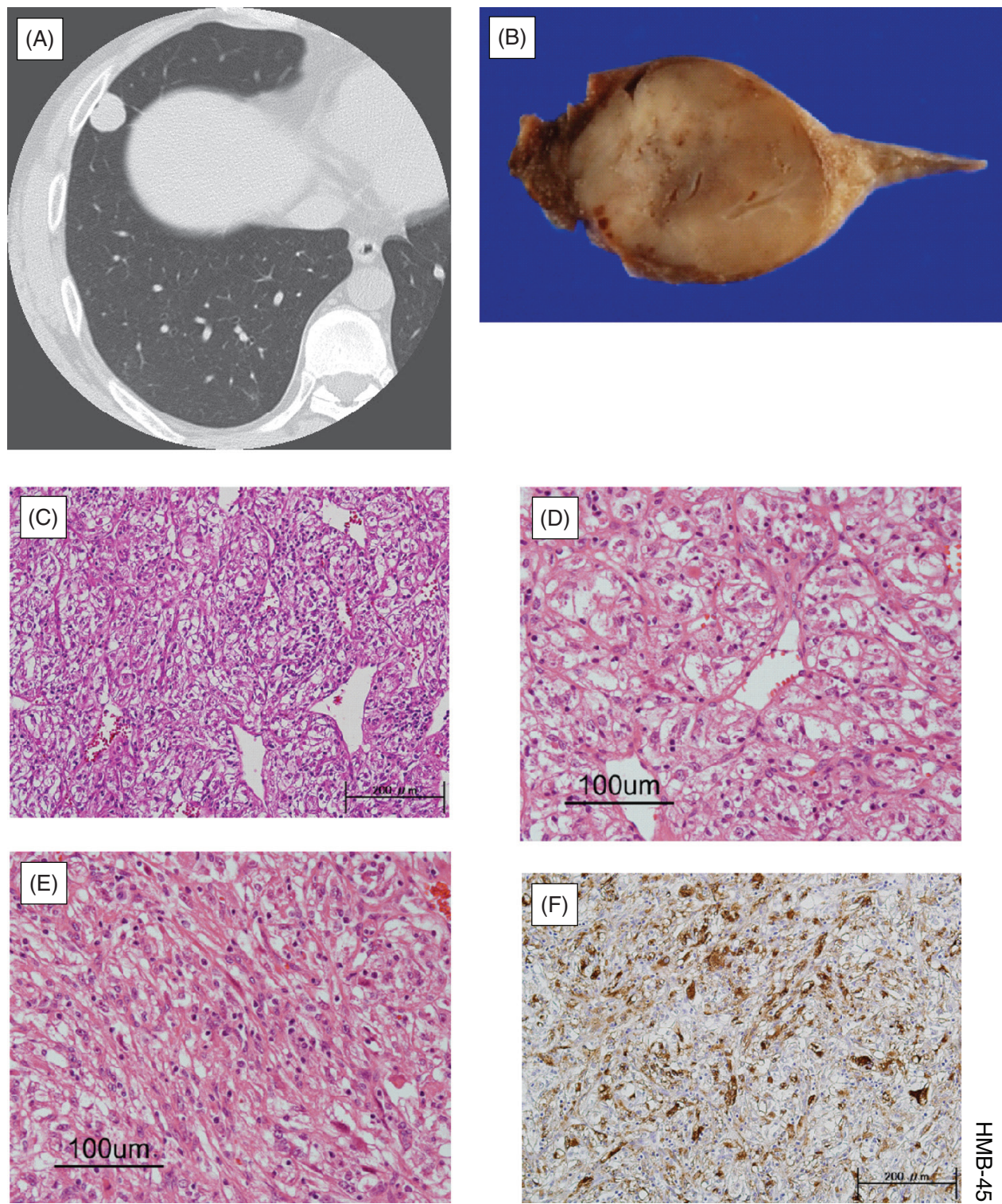

Fig. 1 (A) Tumor is located in the right middle lobe on high-resolution CT. (B) Macroscopically, the tumor contains a solitary, circumscribed and non-capsulated nodule. (C) Histopathological features were consistent with clear cell tumor of the lung (HE stain; original magnification $\times 200$ ). (D) Tumor cells consisted of epithelioid cells, round and oval, with abundant clear to pale eosinophilic cytoplasm $($ HE stain; $\times 400)$. (E) Tumor cells consisted of spindle cells (HE stain; $\times 400)$. $($ F $)$ The tumor cells were positive for HMB-45 $(\times 200)$. CT: computed tomography; HE: hematoxylin and eosin; HMB-45: human melanoma black-45

malignant tumor was suspected. Therefore, he was referred to our hospital for further investigation of the tumor. We scheduled surgery and successfully performed wedge resection of the right middle lobe. The intraoperative frozen section diagnosis was epithelioid tumor without malignancy. The postoperative histological diagnosis was CCT. His postoperative course was uneventful, and he has had no recurrence 13 months after lung resection.
Macroscopically, the wedge resection specimen contained a solitary, circumscribed and non-capsulated nodule (Fig. 1B). The tumor measured $1.8 \times 1.5 \times 1.3 \mathrm{~cm}$. On microscopic examination, the tumor cells consisted predominantly of epithelioid cells, which were round and oval with abundant clear to pale eosinophilic cytoplasm (Fig. 1C, 1D, and 1E). The tumor cells also demonstrated spindle cells, and we observed many dilated thinwalled sinusoidal vessels. There was no sign of necrosis 
or mitoses. Lymphovascular invasion was absent. Periodic acid-Schiff (PAS) staining was positive in the cytoplasm of the tumor cells while PAS with diastase digestion staining were negative because of the glycogen-rich cytoplasm.

The round, oval, and spindle-shaped tumor cells were positive for human melanoma black-45 (HMB-45), smooth muscle actin (SMA), and vimentin; and negative for S100, desmin, AE 1/3, epithelial membrane antigen (EMA), and CD117 (Fig. 1F). There was no difference of staining intensity of HMB-45, SMA, and Vimentin between the round, oval, and spindle-shaped tumor cells.

Both CCT and AML are classified in the same category of PEComas. ${ }^{1,2)}$ We reviewed a specimen of a $7.5 \times 5.0 \times$ 4.5-cm hepatic AML resected in 2005. It consisted predominantly of round, oval cells with abundant eosinophilic cytoplasm (Fig. 2A). It also contained adipose tissue and sinusoidal vessels. Moreover, extramedullary hematopoiesis was frequently noted in the tumor. There was no sign of necrosis or mitoses. Lymphovascular invasion was absent. The tumor cells were positive for HMB-45 and vimentin, focally positive for SMA, and negative for S100, desmin, AE 1/3, EMA, and CD117 (Fig. 2B). The CCT of the lung did not demonstrate any adipocytes, whereas the AML of the liver demonstrated some adipocytes.

\section{Discussion}

PEComas commonly show the presence of epithelioid to spindle cells with eosinophilic to clear cytoplasm, and demonstrate positive immunostaining for melanocytic markers, such as HMB-45 and melan-A. ${ }^{1)}$ CCT, AML, and LAM are considered to belong to a family of PEComas. This case showed metachronous CCT of the lung and AML of the liver. Some reports have documented that AML is complicated with LAM and that AML occurs multifocally in the kidney, liver, and lung. ${ }^{4-6)}$ This case is, to our knowledge, the first report of multiple PEComas, a combination of CCT of the lung and AML of the liver. These findings suggest that patients with PEComas may require whole-body follow-up examinations because PEComas may occur multifocally.

There have been recent reports of malignant PEComas. ${ }^{2,4,7)}$ PEComas can be aggressive and can lead to multiple metastases. Malignant PEComas have been shown to have malignant phenotypes, such as necrosis, infiltrative growth patterns, large tumor size, and high mitosis (>1/50 HPF).,7) The size of malignant hepatic
(A)

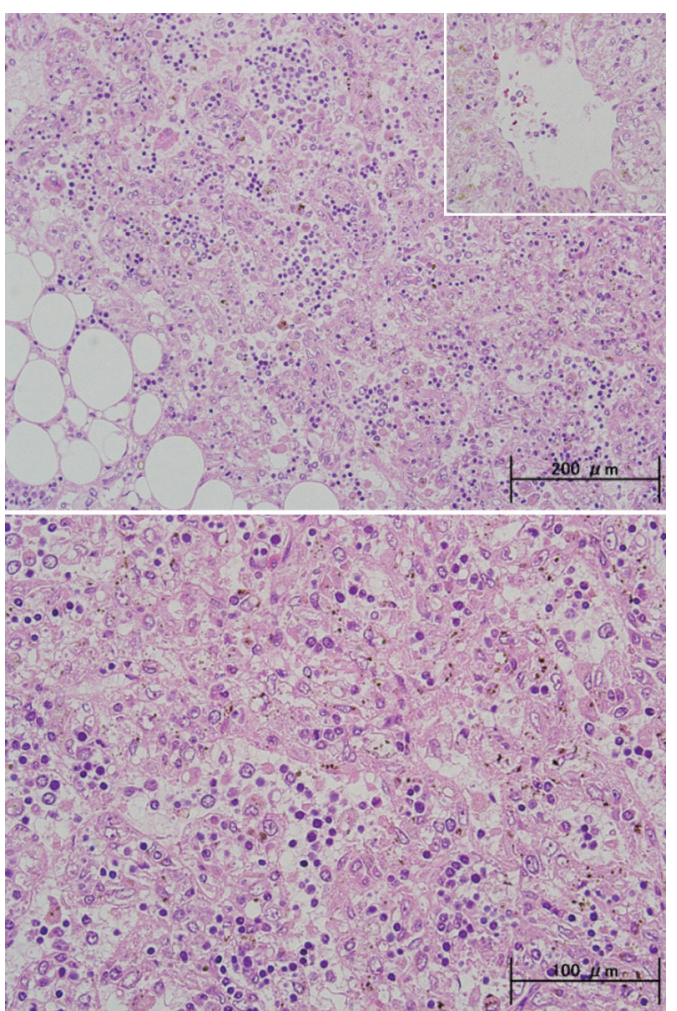

(B)

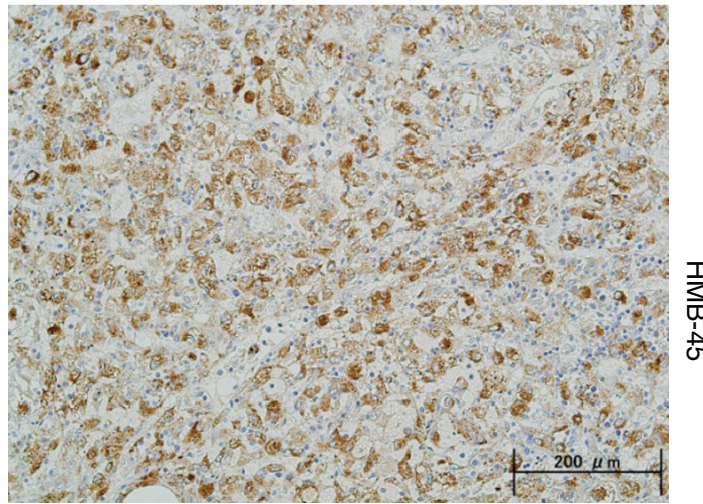

Fig. 2 (A) Histopathological features were consistent with angiomyolipoma (HE stain; original magnification $\times 200$ [upper], $\times 400$ [lower]). (B) Tumor cells were positive for HMB-45 $(\times 400)$.

AML was reported to be greater than $10 \mathrm{~cm} .{ }^{8)} \mathrm{CCT}$ of the lung of greater than $5 \mathrm{~cm}$ in size may be associated with malignancy. ${ }^{9)}$ Aforementioned AML occurred multifocally in the kidney, liver, and lung, had necrosis and mitosis, or was greater than $10 \mathrm{~cm} .{ }^{4,5,10,11)}$

The findings of this case did not match the above criteria for malignancy. These two tumors showed low mitotic activity, and no sign of necrosis. Lymphovascular invasion was not observed. The sizes of the CCT and AML were less than $5 \mathrm{~cm}$ and $10 \mathrm{~cm}$, respectively. In addition, each tumor of the lung and the liver was 
solitary. The CCT did not show any adipocytes, whereas the AML showed some adipocytes. Therefore, the both tumors of the hepatic AML and the pulmonary CCT were not considered to be malignant, and this case demonstrated metachronous multiple PEComas.

It has also been shown that PEComas are involved in tuberous sclerosis (TS), which is an autosomal dominant genetic disease due to losses of TSC1 or TSC2 genes, and that these tumor suppressor genes might play an important role in its pathogenesis. ${ }^{2,12)}$ Some reports have documented that AML and LAM were complications of TS. ${ }^{2)}$ This case showed metachronous CCT of the lung and AML of the liver, but these were not associated with TS. Although we did not perform genetic analysis in this case, further investigation using molecular and genetic techniques is required to better understand the mechanisms of PEComas with/without TS.

Sirolimus, an inhibitor of cytoplasmic protein mammalian target of rapamycin (mTOR), was reported to be an effective drug for LAM. ${ }^{13)}$ A previous report describes 2 patients with AML who were treated with sirolimus or temsirolimus, a sirolimus prodrug. ${ }^{14)}$ It is possible that malignant or multiple PEComas can be treated with the same drugs.

\section{Conclusion}

We report a case with metachronous double PEComas of a combination of CCT of the lung and AML of the liver. The findings of the current study suggest that patients with PEComas may require whole-body follow-up examinations because different subtypes of PEComas may occur multifocally.

\section{Disclosure Statement}

The authors have reported that no potential conflicts of interest exist with any companies/organizations whose products or services may be discussed in this article.

\section{References}

1) Fletcher CDM, Unni KK, Mertens F. Neoplasms with perivascular epithelioid cell differentiation (PEComas). Pathology and Genetics of Tumours of Soft Tissue and Bone. Lyon: IARC Press, 2002; pp221-22.

2) Martignoni G, Pea M, Reghellin D, et al. PEComas: the past, the present and the future. Virchows Arch 2008; 452: 119-32.

3) Bonetti F, Pea M, Martignoni G, et al. PEC and sugar. Am J Surg Pathol 1992; 16: 307-8.

4) Saito M, Tsukamoto T, Takahashi T, et al. Multifocal angiomyolipoma affecting the liver and lung without tuberous sclerosis. J Clin Pathol 2004; 57: 221-4.

5) Eble JN. Angiomyolipoma of kidney. Semin Diagn Pathol 1998; 15: 21-40.

6) Mizushina Y, Bando M, Hosono T, et al. Clinical features of lymphangioleiomyomatosis complicated by renal angiomyolipomas. Intern Med 2011; 50: 285-9.

7) Folpe AL, Mentzel T, Lehr HA, et al. Perivascular epithelioid cell neoplasms of soft tissue and gynecologic origin: a clinicopathologic study of 26 cases and review of the literature. Am J Surg Pathol 2005; 29: 1558-75.

8) Nguyen TT, Gorman B, Shields D, et al. Malignant hepatic angiomyolipoma: report of a case and review of literature. Am J Surg Pathol 2008; 32: 793-8.

9) Ye T, Chen H, Hu H, et al. Malignant clear cell sugar tumor of the lung: patient case report. J Clin Oncol 2010; 28: e626-8.

10) Cibas ES, Goss GA, Kulke MH, et al. Malignant epithelioid angiomyolipoma ('sarcoma ex angiomyolipoma') of the kidney: a case report and review of the literature. Am J Surg Pathol 2001; 25: 121-6.

11) Varma $S$, Gupta $S$, Talwar J, et al. Renal epithelioid angiomyolipoma: a malignant disease. J Nephrol 2011; 24: 18-22.

12) Crino PB, Nathanson KL, Henske EP. The tuberous sclerosis complex. N Engl J Med 2006; 355: 1345-56.

13) McCormack FX, Inoue Y, Moss J, et al. Efficacy and safety of sirolimus in lymphangioleiomyomatosis. N Engl J Med 2011; 364: 1595-606.

14) Wolff N, Kabbani W, Bradley T, et al. Sirolimus and temsirolimus for epithelioid angiomyolipoma. J Clin Oncol 2010; 28: e65-8. 